\title{
Designing Integrated Silicon Photonics Flexible High-Data Rate Transceivers (NNX16AD14G-Y1)
}

\author{
SHAYAN MOOKHERJEA \\ Department of Electrical and Computer Engineering, University of California, San Diego, MC 0407 La \\ Jolla CA 92093-0407 USA \\ Email:smookher@ucsd.edu
}

We study a transceiver architecture, which is based on an innovative design which generates a flexible number of communication streams from a single laser. This approach can achieve reductions in size, weight, and energy consumption, and improvements on link performance and bandwidth compared to both RF communications and existing optical technologies Summary of a Project Outcomes report of research funded by NASA.

\section{Introduction and Goals}

The objective of this project is to develop the technology for silicon photonic transceiver components that can comprise a coherent laser optical communication and/or relay network in near-Earth space at wavelengths near $1550 \mathrm{~nm}$, for use on a satellite platform. Our proposed transceiver architecture, which is based on an innovative design which generates a flexible number of communication streams from a single laser, offers scalable very high bit-rate communications with orders-of-magnitude reductions in size, weight, and energy consumption, and improvements on link performance and bandwidth compared to both RF communications and existing optical technologies. Our technical approach involves an innovative design to the components, fabrication of integrated chipsets using a silicon foundry process and testing at the breadboard level in support of future monolithic and heterogeneous integration.

\section{Activities}

The complete device consists of three building blocks: the recirculating variable-count frequency shifter (RVCFS), the high-order modulation format encoder, and the detector. Of these, the second and third are fairly standard, though complex, components in modern silicon photonics, and the major risk (as well as most of the rewards in terms of energy, cost, and weight savings) in this project is carried by the first component.

We have started and made substantial progress in creating a working model of the RVCFS component in an industry-standard software package (Lumerical Interconnect), which validates the concept in the frequency domain. The recirculating variable-count frequency shifter (RVCFS) component itself comprises of a single-sideband carrier-suppressed (SSBCS) modulator driven by an externally-supplied RF tone, a tunable optical bandpass filter, and 
(ideally) a gain section i.e., optical amplifier, which are placed in a loop as shown in the figure below. Key innovations are the way new frequency tones are generated (essentially, a nonlinear process) with an even spacing, and how the number of tones is controlled by programmable optical bandpass filtering.

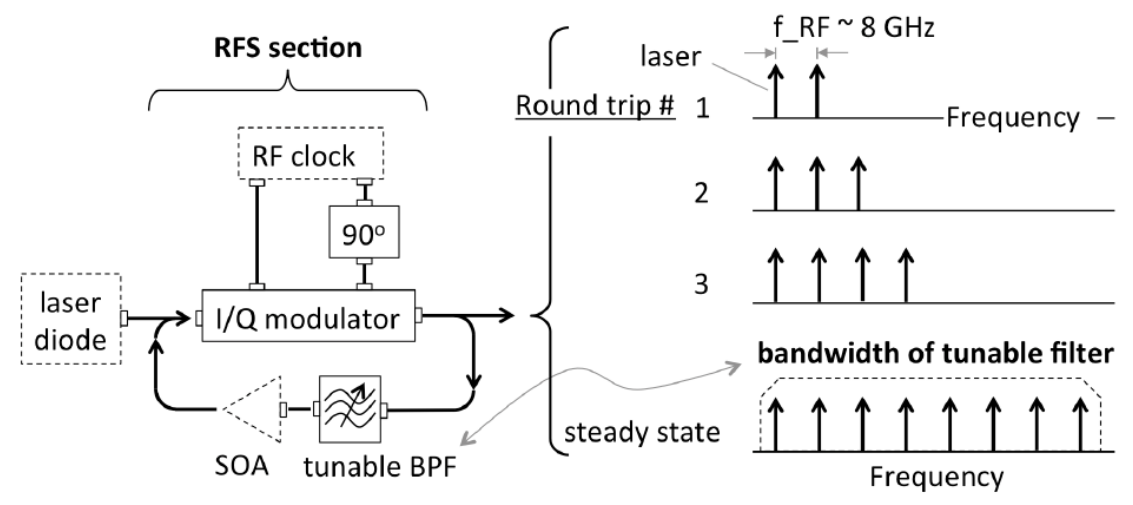

Figure 1 Key innovative component: recirculating variable-count frequency shifter (RVCFS) component, which generates a variable number of spectral tones from a single $\mathrm{CW}$ laser diode

Prior to this project, we have studied optical wave propagation, bandpass filtering and wavelength conversion (for the generation of new wavelengths) using the silicon coupled-resonator optical waveguide structure[1]-[4], which has a resonantly enhanced nonlinearity but uses relatively low quality-factor resonators coupled in a serial cascade [5]. As an example, we have demonstrated the time-energy entangled nature of the photon pairs generated by our devices around $1550 \mathrm{~nm}[6]$, thus showing that standard silicon photonics foundry processing can be used to make high performance devices with stringent precision requirements. However, such higher-order filtering devices are inherently sensitive to fabrication disorder and a simpler reducedcomplexity structure may be optimal. A simpler I/Q modulator formed using a couple of phase shifter waveguides or microring filters may be easier to control and operate. We have studied and made improvements in monitoring and stabilizing a microresonator [7].

In preparing designs for fabrication, we have created, and then used, a Lumerical Interconnect model to model the first part of this loop, and studied the impact of fabrication imperfections in the key parameters: couplingcoefficient error (e.g., deviation from 50-50 split ratio), path length mismatch, deviation from linearity in the phase shifter etc. This has also required some abinitio studies of optics and electronics using the Mode, FDTD and Device software components, as shown in Figure 2.

Most of our design activities during this period were focused on the upcoming chip tapeout with Sandia National Labs. This US-based foundry has a fabrication process supporting Si photonic actives (tuned for a specific kind of $\mathrm{p}-\mathrm{n}$ junction) and passives (similar to comparable foundries like IME, 
Singapore and IMEC Belgium), $\mathrm{Si}_{3} \mathrm{~N}_{4}$ passives (including low-loss couplers) and Ge photodetectors (superior to other foundries, especially in terms of performance at low signal levels, which will benefit this project). We have worked with the Sandia process before this and have a high degree of confidence in being able to yield functional chips, but are aware of the complexities and challenges of this design. Therefore, the project plan includes a two-stage chip-fabrication effort, wherein the first stage is dedicated to test structures and the second stage is dedicated to more complete functional blocks.

\section{Impact}

There are several couplers in the architecture which must be carefully designed, fabricated, and potentially trimmed after fabrication. One of the advantages of the integrated device architecture is that directional couplers are well controlled, although the dispersion (i.e., wavelength sensitivity) of the directional coupler can be large. The same large dispersion issue also affects the coupled-resonator device and can sometimes be used for added functionality[8], [9]. As an alternative, we can use multi-mode interference (MMI) couplers.

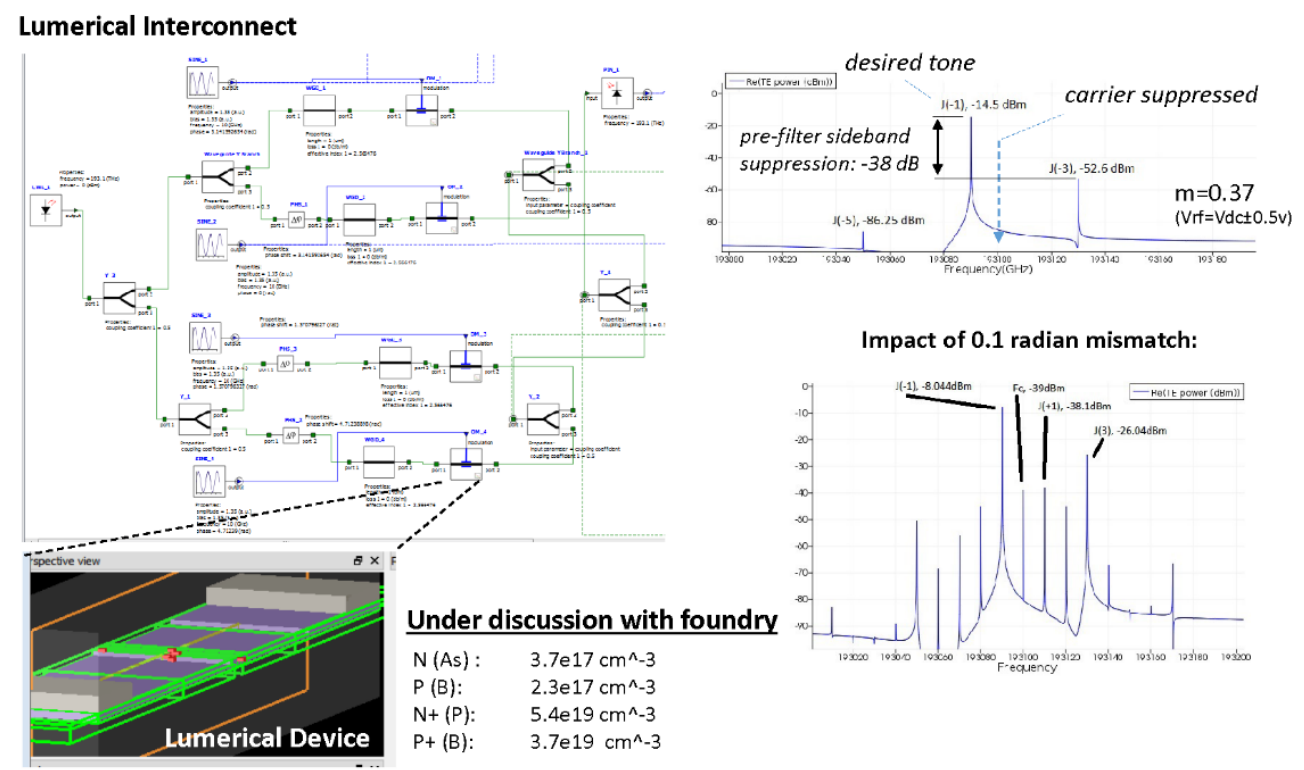

Figure 2 Modeling of the SSBSC modulator and investigations of tolerances was performed before mask generation and fabrication.

Based on the detailed modeling, we can study the impact of different types of optical filters on the predicted performance. For example, Fig. 3 shows the differences between perfect 'rect' filters (which are unrealizable in practice) and more-realistic Gaussian filters when generating a number of tones in the RVCFS loop architecture. A conservative set of assumptions including typical 
errors and imbalances was used in this model: RF phase between push/pull MZM drivers offset by 0.5 radians (error); optical phase shift (heaters) mismatched by 0.1 radians and 0.2 radians (top and lower arm) i.e., effectively equaling $10 \mathrm{~nm}$ and $19 \mathrm{~nm}$ of path-length difference; RF phase noise: $-60 \mathrm{dBc}$ close-in; $-135 \mathrm{dBbc}$ wideband + AWGN floor at $-135 \mathrm{dBm}$; Laser seed with $12.5 \mathrm{MHz}$ linewidth, $0 \mathrm{dBm}$ input; SOA spec'd at $7.9 \mathrm{~dB}$ ASE noise, $500 \mathrm{~mA}$ input, $P_{-}$sat $=17 \mathrm{dBm}$. These simulations show that non-ideal filters can result in some extra sidebands outside the target passband, but the peak-to-sideband ratio can be reduced by increasing the filter order (at the cost of device complexity).

The work of one graduate student at UCSD was supported by this project. The student received training in the fields of electrical engineering, physics, device fabrication and measurement. Results have been published in peer-reviewed journals and presented at internationally-recognized conferences, as well as workshops, seminars, and meetings.
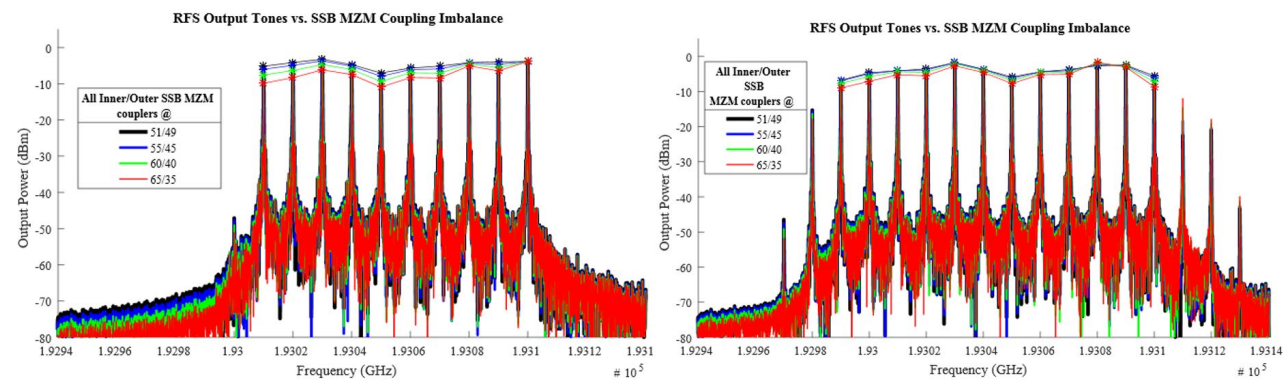

Figure 3 Simulated effect of higher-order optical filter roll-off on the extinction of out-of-band tones in the recirculating loop. Perfect Rect filter shape (left) vs. Cascaded Gaussian filter (right).

The recirculating loop needs a single-sideband modulator, which can be formed using conventional Mach-Zehnder interferometer structures and a tunable front-end for balancing the phase. A coherent I/Q modulator uses a related structure, as indicated in Figure 4. Four MZMs are arranged in an IQ superstructure, with two cascaded in the upper arm (the in-phase "I" arm), and two cascaded in the lower arm (the quadrature " $Q$ " arm). The modulator was designed to be driven using binary electrical signals, which is easier than generating multilevel signals. Only one electrical tuning knob is needed to control for phase drift between the arms. 


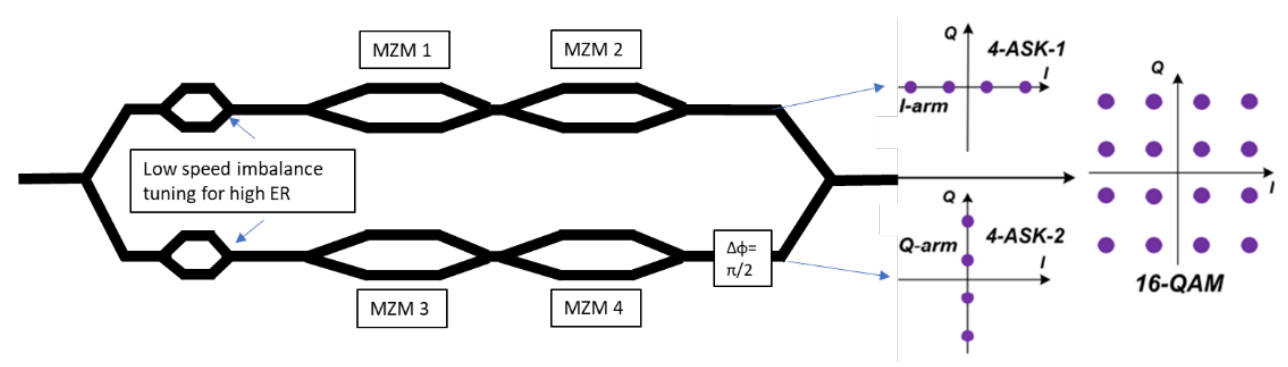

Figure 4 Schematic of a coherent communications modulator (I/Q modulator).

\section{Potential Subsequent Extensions}

We use conventional silicon photonics for the electro-optic modulator. However, an alternative emerging technology, which integrates thin-film lithium niobate (TFLN) with silicon photonic passive circuits, offers ultrahigh modulation bandwidth that is beyond the capabilities of traditional depletionmode Mach-Zehnder modulators [10]. Although these devices cannot be fully made in a silicon microfabrication facility (due to the inclusion of lithium niobate), the template structures necessary for light confinement can be fully made in a standard process, and TFLN can be incorporated by bonding as an unetched, un-patterned thin film only in the regions where it is needed. In the future, an integrated process flow may be realized, which will help designers extend the bandwidth of integrated electro-optic phase modulators beyond 100 $\mathrm{GHz}$.

One of the challenges of high index contrast integrated photonics is the sensitivity of resonant structures to disorder. This can be seen in the coupledresonator configuration which can sometimes use a large number of microresonators [11], [12] and affects the extinction ratio of filters using this type of structure. However, we have also studied ways to study and potentially compensate for disorder after fabrication [13]-[15]. Filters used as part of a recirculating loop have relatively modest requirements, since optical gain selfselects the strongest tones in the loop and a small amount of contrast in a single pass usually results in a much stronger contrast in the steady state. However, certain communications systems which use coherent modulation formats have stringent requirements on linewidth and out of band signal extinction, and filter performance should be improved as much as possible.

All recirculating loop devices, especially those with gain in the loop, have to eventually consider the effects of nonlinearity as the optical power is increased. Physics theory suggests that there are other interesting Kerr nonlinear effects that might occur in appropriately dispersion-engineered devices, such as ways to prevent the spreading of light through soliton generation [16]. However, these effects are mostly beyond the scope of this project. 


\section{Open-Access Reporting Initiative}

PRAISE: This open-access document is provided in support of our PRAISE (Public Report of Activities, Impact and Subsequent Extensions) initiative. What is it? An open-access document shared with the public which describes the research outcomes of publicly-funded projects such as those funded by the U.S. NSF (National Science Foundation) and other agencies.

\section{References}

[1] S. Mookherjea, "Spectral characteristics of coupled resonators," J. Opt. Soc. Am. B, vol. 23, no. 6, p. 1137, Jun. 2006, doi: 10.1364/JOSAB.23.001137.

[2] S. Mookherjea, D. S. Cohen, and A. Yariv, "Nonlinear dispersion in a coupled-resonator optical waveguide," Opt. Lett., vol. 27, no. 11, p. 933, Jun. 2002, doi: 10.1364/OL.27.000933.

[3] J. R. Ong and S. Mookherjea, "Quantum light generation on a silicon chip using waveguides and resonators," Opt. Express, vol. 21, no. 4, p. 5171, Feb. 2013, doi: 10.1364/OE.21.005171.

[4] M. L. Cooper et al., "235-ring Coupled-Resonator Optical Waveguides," in Conference on Lasers and Electro-Optics 2010, San Jose, California, 2010, p. CTUHH3. doi: 10.1364/CLEO.2010.CTuHH3.

[5] J. R. Ong, R. Kumar, and S. Mookherjea, "Silicon microring-based wavelength converter with integrated pump and signal suppression," Opt. Lett., vol. 39, no. 15, p. 4439, Aug. 2014, doi: 10.1364/OL.39.004439.

[6] R. Kumar, M. Savanier, J. R. Ong, and S. Mookherjea, "Entanglement measurement of a coupled silicon microring photon pair source," Opt. Express, vol. 23, no. 15, p. 19318, Jul. 2015, doi: 10.1364/OE.23.019318.

[7] M. Savanier, R. Kumar, and S. Mookherjea, "Optimizing photon-pair generation electronically using a $p-i-n$ diode incorporated in a silicon microring resonator," Appl. Phys. Lett., vol. 107, no. 13, p. 131101, Sep. 2015, doi: 10.1063/1.4932047.

[8] S. Mookherjea, "Using gain to tune the dispersion relation of coupledresonator optical waveguides," IEEE Photon. Technol. Lett., vol. 18, no. 5, pp. 715-717, Mar. 2006, doi: 10.1109/LPT.2006.871144.

[9] S. Mookherjea, "Semiconductor coupled-resonator optical waveguide laser," Appl. Phys. Lett., vol. 84, no. 17, pp. 3265-3267, Apr. 2004, doi: 10.1063/1.1719278.

[10] X. Wang, P. O. Weigel, J. Zhao, M. Ruesing, and S. Mookherjea, "Achieving beyond-100-GHz large-signal modulation bandwidth in hybrid silicon photonics Mach Zehnder modulators using thin film lithium niobate," APL Photonics, vol. 4, no. 9, p. 096101, Sep. 2019, doi: 10.1063/1.5115243. 
[11] M. L. Cooper and S. Mookherjea, "Modeling of Multiband Transmission in Long Silicon Coupled-Resonator Optical Waveguides," IEEE Photon. Technol. Lett., vol. 23, no. 13, pp. 872-874, Jul. 2011, doi: 10.1109/LPT.2011.2141657.

[12] S. Mookherjea and M. A. Schneider, "Avoiding bandwidth collapse in long chains of coupled optical microresonators," Opt. Lett., vol. 36, no. 23, p. 4557, Dec. 2011, doi: 10.1364/OL.36.004557.

[13] M. L. Cooper, G. Gupta, J. S. Park, M. A. Schneider, I. B. Divliansky, and S. Mookherjea, "Quantitative infrared imaging of silicon-on-insulator microring resonators," Opt. Lett., vol. 35, no. 5, p. 784, Mar. 2010, doi: 10.1364/OL.35.000784.

[14] S. Mookherjea and H. R. Grant, "High dynamic range microscope infrared imaging of silicon nanophotonic devices," Opt. Lett., vol. 37, no. 22, p. 4705, Nov. 2012, doi: 10.1364/OL.37.004705.

[15] Y. Shen, I. B. Divliansky, D. N. Basov, and S. Mookherjea, "Perfect set-and-forget alignment of silicon photonic resonators and interferometers," in Optical Fiber Communication Conference/National Fiber Optic Engineers Conference 2011, Los Angeles, California, 2011, p. PDPC3. doi: 10.1364/OFC.2011.PDPC3.

[16] B. Crosignani, A. Yariv, and S. Mookherjea, "Nonparaxial spatial solitons and propagation-invariant pattern solutions in optical Kerr media," Opt. Lett., vol. 29, no. 11, p. 1254, Jun. 2004, doi: 10.1364/OL.29.001254. 